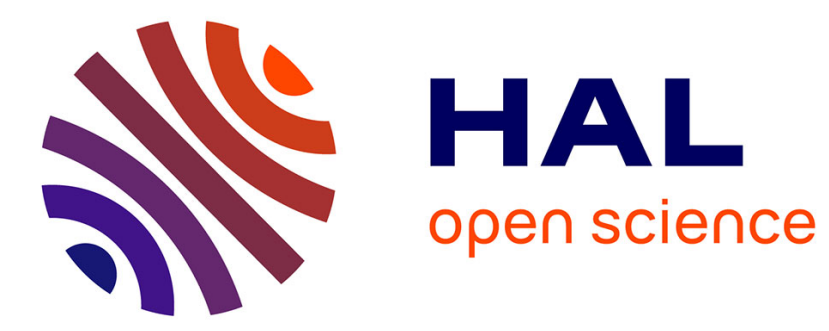

\title{
Recherches photométriques sur les flammes colorée M. Gouy
}

\section{- To cite this version:}

M. Gouy. Recherches photométriques sur les flammes colorée. J. Phys. Theor. Appl., 1880, 9 (1), pp.19-27. 10.1051/jphystap:01880009001901 . jpa-00237636

\section{HAL Id: jpa-00237636 https://hal.science/jpa-00237636}

Submitted on 1 Jan 1880

HAL is a multi-disciplinary open access archive for the deposit and dissemination of scientific research documents, whether they are published or not. The documents may come from teaching and research institutions in France or abroad, or from public or private research centers.
L'archive ouverte pluridisciplinaire HAL, est destinée au dépôt et à la diffusion de documents scientifiques de niveau recherche, publiés ou non, émanant des établissements d'enseignement et de recherche français ou étrangers, des laboratoires publics ou privés. 


\section{REGHERGHES PHOTOMÉTRIQUES SUR LES FLAMMES COLORÉES;}

Par M. GOUY.

Les propriétés optiques des vapeurs métalliques incandescentes ont été l'objet d'un grand nombre de travaux, dont quelques-uns 
sont devenus classiques, et qui ont eu pour objet principal la description exacte des spectres donnés par les vapeurs et les gaz incandescents. Il en résulte que l'analyse spectrale, envisagée au point de vue chimique, est aujourd'hui établie sur des bases solides et permet, en étudiant le rayonnement du Soleil ou d'une étoile, de reconnaître la plupart des substances qui forment la surface incandescente. Mais il n'en est pas de même de l'état physique de ces substances et, en l'absence de données sur ce point, il est impossible actuellement de reconnaitre la température, l'épaisseur et la densité d'une couche de vapeur par l'étude de son rayonnement.

En effet, nous ne savons rien de précis sur l'influence de la densité et de la température. Quant à l'épaisseur, la théorie nous apprend que l'intensité d'un rayon simple est proportionnelle à I $-e^{-m \mathrm{E}}, \mathrm{E}$ étant l'épaisseur de la couche, supposée homogène, et $m$ un coefficient qui dépend de la longueur d'onde. Mais cette formule n'est pas immédiatement applicable. En effet, il est facile de démontrer qu'une raie ne peut jamais être composée d'un rayon simple ou de longueur d'onde unique, mais résulte nécessairement du groupement d'une infinité de rayons voisins (1); en d'autres termes, que l'intensité du rayonnement est une fonction continue de la longueur d'onde $\lambda$, fonction dont le maximum forme la raie. Dès lors, l'intensité de la raie est proportionnelle à l'intégrale $\int\left(1-e^{-m \mathrm{E}}\right) d \lambda$, prise entre des limites convenables; la variation de l'intensité avec $\mathrm{E}$ dépend donc de la fonction inconnue qui lie $m$ à $\lambda$. Ces diverses questions exigeaient donc de nouvelles recherches, et il était nécessaire de procéder à des mesures photométriques.

I. Appareil. - L'appareil que j'ai employé pour ces mesures a la forme générale d'un spectroscope à deux prismes ( $f i g$. 1$)$. Le collimateur $\mathrm{C}$ a son objectif à demi recouvert par un miroir plan $\mathrm{M}$,

( ${ }^{1}$ ) On trouvera cette démonstration, avec d'autres détails que je ne puis donner ici, dans un Mémoire publié dans les Annales de Chimie et de Physique, $5^{\circ}$ série, t. XVIII, p. 5. Je profite de l'occasion pour rectifier, dans cette démonstration, un lapsus d'ailleurs facile à reconnaître : il y est dit, page 10, qu'un élément infiniment petit $\omega$ reçoit une quantité finie de lumière, ce qui n'a pas de sens; l'élément $\omega$ doit être regardé comme ayant une surface très petite, mais déterminée. 
sur lequel se réfléchissent les rayons sortis d'un second collimateur $\mathrm{C}^{\prime}$; ces rayons, après réflexion, deviennent parallèles aux rayons

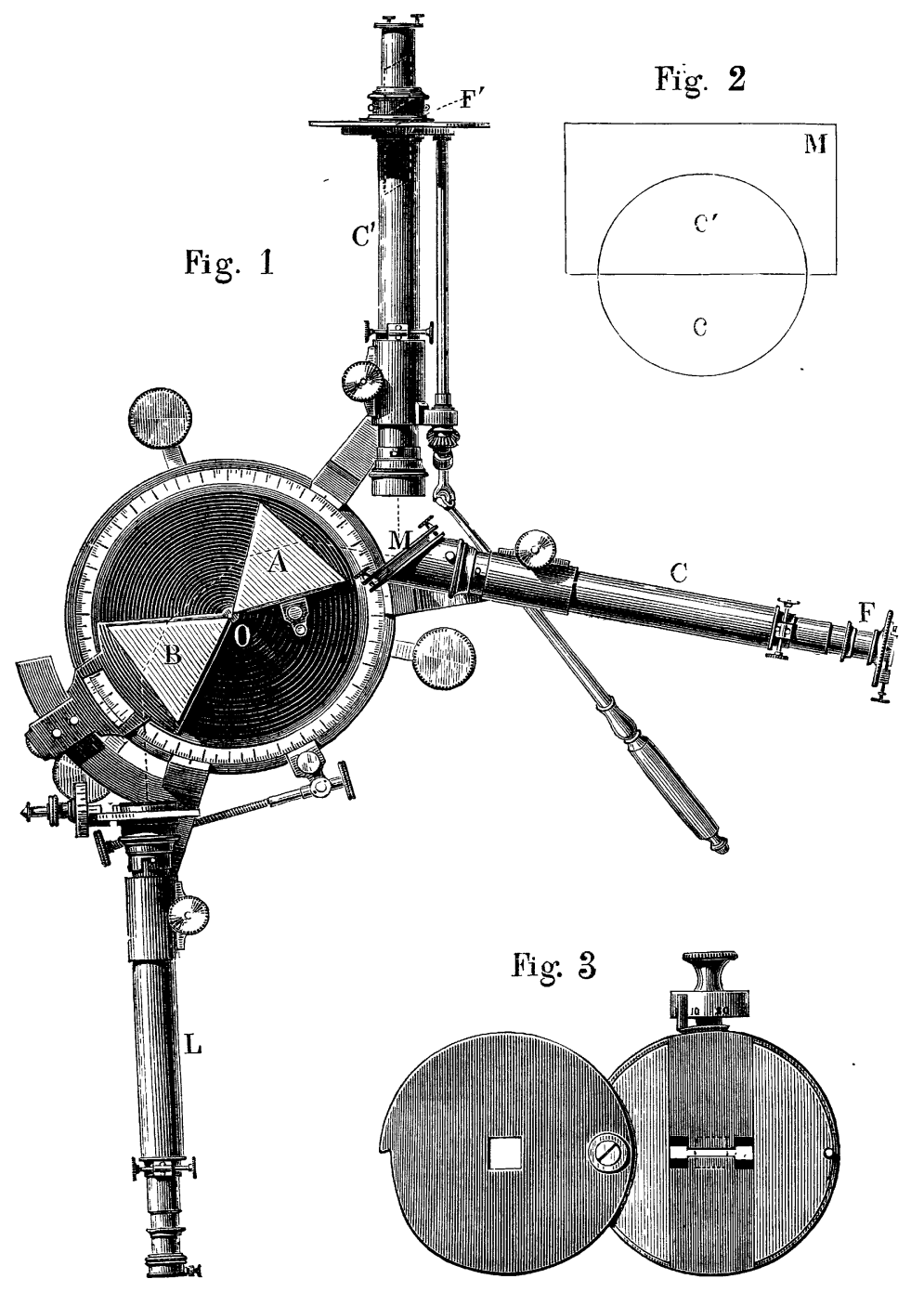

sortis de C, et, par suite, au foyer de la lunette L, nous aurons deux spectres en coïncidence exacte, fournis l'un par C, l'autre 
par $\mathrm{C}^{\prime}$. Ce dernier spectre est continu et donné par une lampe à gaz; le spectre donné par $\mathrm{C}$ est, au contraire, un spectre de raies, fourni par la flamme colorée que l'on place devant la fente $\mathbf{F}$. La lunette L, au lieu d'oculaire, porte à son foyer une fente verticale qui ne laisse passer qu'une portion très limitée du spectre ( $f \mathrm{~g} g .3)$. L'œil placé derrière cette fente voit, à travers les prismes, la moitié inférieure de l'objectif de $\mathrm{C}$, et, réfléchie sur le miroir, la moitié supérieure de l'objectif de $\mathrm{C}^{\prime}$, sous l'aspect de deux demi-cercles lumineux de même nuance, $c$ et $c^{\prime}(f i g \cdot 2)$. On peut leur donner le même éclat en faisant varier l'éclat de $c^{\prime}$ au moyen de deux nicols dont est muni le collimateur $C^{\prime}$. L'angle des deux nicols donne les éléments de la mesure photométrique.

Les flammes colorées employées dans ces expériences devaient, pour donner des résultats utiles, être homogènes et d'éclat constant. Pour réaliser ces deux conditions, j’ai employé la méthode suivante. De l'air comprimé passe dans un pulvérisateur et réduit en poussière une solution saline convenablement choisie. Cet air, chargé de poussière saline, se mélange au gaz d'éclairage dans un appareil régulateur, et le mélange, homogène et de proportions constantes, vient brûler dans des lampes formées d'un simple tube cylindrique. Au besoin, cette flamme peut être enfermée dans une autre flamme incolore de même température, pour la préserver de l'influence de l'air extérieur.

II. Transparence des flammes pour les divers rayons. - Nous allons maintenant passer en revue les recherches faites avec ces appareils.

$a$. Je me suis occupé tout d'abord de la transparence des flammes colorées, et, en premier lieu, de leur transparence pour les rayons qu'elles n'émettent pas ou n'émettent qu'en petite quantité; pour cela, je faisais traverser la flamme par le rayonnement d'une lampe à gaz. L'absorption a toujours été trouvée insensible : ce résultat est conforme à la relation connue entre les pouvoirs émissif et absorbant.

b. J'ai abordé ensuite l'étude de la transparence de la flamme pour les radiations qu'elle émet elle-même; pour cela, j'ai fait passer à travers la flamme les rayons émis par une autre flamme de même espèce et de même température. Mes premiers essais m'ont montré 
que, dans le cas où il y a une absorption sensible, cette absorption dépend à la fois de l'éclat de la flamme qui rayonne et de celui de la flamme qui est traversée par les rayons, en sorte que, à température constante, l'absorption dépend de deux variables. Cette complexité du phénomène, qui serait incompréhensible si les raies étaient formées de lumière homogène, m'a obligé à me borner d'abord à l'étude d'un cas particulier, qui permet, comme nous le verrons plus loin, de traiter le problème général. Ce cas particulier est celui où nos deux flammes sont identiques.

III. Cas des flanmes identiques. - Ces deux flammes sont produites par le même mélange et dans des lampes identiques; on s'assure directement qu'elles ont le même éclat. Cela fait, on mesure l'éclat $i$ d'une raie donnée par l'une d'elles et l'éclat $i^{\prime}$ de la mème raie donnée par les deux flammes réunies, rayonnant l'une à travers l'autre. C'est le rapport $\frac{i^{\prime}}{i}$, que nous désignerons par la lettre $k$, qui exprime le résultat de l'expérience; c'est donc le rapport suivant lequel s'accroît l'éclat de la raie quand on double l'épaisseur de la couche homogène de vapeur.

Pour les bandes larges et les spectres continus, on a sensiblement $k=2$ avec les flammes les plus chargées que j'aie pu produire; par suite, la transparence est ici complète.

Pour les raies étroites, $k$ est variable avec l'éclat $i$ de la raie donnée par une seule flamme, éclat qu'on fait varier en chargeant la flamme de quantités variables de vapeurs métalliques. Pour des flammes très peu chargées, $k$ est égal à 2 ; l'éclat augmentant, il diminue, passe par un minimum, puis par un maximum peu différent, et devient ensuite sensiblement constant jusqu'à la limite des expériences. Voici les valeurs du minimum pour les diverses raies étroites :

Potassium, raie rouge........ I , 47

Rubidium, raie violette $(\lambda=42.02) \ldots \quad \mathrm{r}, 42$

Sodium, raie jaune.......... I, 38

Strontium, raie bleue......... I, 34

Lithium, raie rouge......... I , 32

Calcium, raie violette......... I ,25 environ.

Pour le sodium, ces résultats se rapportent à l'ensemble des deux 
raies $D_{1}$ et $D_{2}$. Si l'on opère sur chaque raie séparément, on trouve que dans une même flamme les deux raies, qui ont toujours un éclat différent, donnent pour $k$ des valcurs différentes, mais que néanmoins pour le même éclat elles donnent la même valeur de $k$. Les deux raies violettes du rubidium donnent lieu à la même remarque. Nous verrons plus loin comment, au moyen de ces résultats, nous traiterons le problème général, et nous allons nous occuper de la densité de la vapeur métallique.

IV. Loi du rayonnement. - Considérons une couche homogène de vapeur, d'épaisseur $\mathrm{E}$; soit $p$ le pouvoir émissif de la couche pour un rayon de longueur d'onde déterminée. Si $\mathbf{E}$ prend un accroissement infiniment petit $d \mathbf{E}$, l'accroissement correspondant $d p$ peut se calculer ainsi. Le pouvoir émissif de la couche d'épaisseur $d \mathrm{E}$ qui s'ajoute à la couche primitive est $m \mathrm{D} d \mathrm{E}$, D étant la densité de la vapeur, et $m$ un coefficient constant (à une mème température). Les rayons partis de cette couche traversent la couche primitive, dont le pouvoir absorbant est $p ;$ il vient donc

$$
d p=m \mathbf{D}(\mathbf{r}-p) d \mathbf{E}
$$

dont l'intégrale est $p=\mathrm{I}-\mathrm{C} e^{-m \mathrm{DE}}$.

Comme pour $\mathrm{E}=\mathrm{o}$ on a $p=\mathrm{o}$, la constante $\mathrm{C}$ est égale à l'unité, et il vient

$$
p=1-e^{-m \mathrm{DE}} .
$$

Ainsi, pour chaque rayon simple, le pouvoir émissif et le pouvoir absorbant ne dépendent que du produit DE, qui représente la quantité de vapeur contenue dans un cylindre droit ayant pour base l'unité et pour hauteur l'épaisseur de la couche. Nous désignerons ce produit par la lettre $q$. Ainsi, les propriétés optiques d'une couche homogène de vapeur ne dépendent que de $q$.

Réciproquement, deux couches de vapeur de même température qui donnent le même éclat à une raie donnent pour $q$ le même nombre et sont identiques au point de vue optique. Ces deux couches doivent donner pour $k$ la même valeur; par suite, $k$ n'est fonction que de l'éclat de la raie et doit avoir la même valeur pour 
deux flammes d'épaisseurs différentes, mais de même éclat et de même température.

Ce résultat a été vérifié par l'expérience avec beaucoup d'exactitude pour les trois raies du sodium, du lithium et du strontium.

Voici le tableau de quelques expériences faites sur la raie $\mathbf{D}$ du sodium :

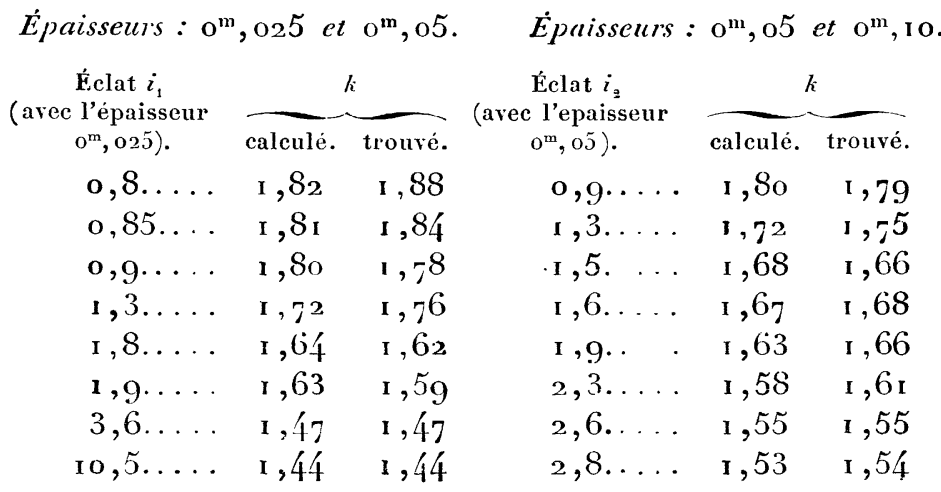

Il est à remarquer que la formule (I) établie dans ce paragraphe a été trouvée en admettant que le rayonnement des atomes est indépendant de leur distance moyenne. Le rayonnement de chacun d'eux est donc indépendant de la densité de la couche de vapeur, ainsi que nous en avons fait l'hypothèse; ce résultat sera confirmé par les expériences suivantes.

V. Conséquences. - D'autre part, la fonction qui lie l'éclat $i$ de la raie au produit $q$ peut se calculer aisément dans notre hypothèse. En effet, pour $i=1$, prenons arbitrairement $q=\mathbf{1}$; pour $q=2$, nous aurons $i=k_{1}$, en appelant $k_{1}$ la valeur de $k$ qui correspond à $i=\mathrm{r}$. Soit $k_{2}$ la valeur de $k$ qui correspond à $i=k_{1}$; pour $q=4$, nous aurons $i=k_{1} k_{2}$, et ainsi de suite. On peut aussi calculer les valeurs de $i$ pour les valeurs de $q$ qui ne se trouvent pas comprises dans la progression géométrique précédente. Voici quelques valeurs de cette fonction, calculées pour la raie jaune du sodium :

$\begin{array}{ll}q . & i . \\ \mathbf{I} & \mathbf{I} \\ \mathbf{2} & \mathbf{I}, 9^{\mathbf{0}}\end{array}$




\begin{tabular}{cr}
$q$. & \multicolumn{1}{c}{$i}$. \\
5 & 4,09 \\
10 & 6,66 \\
100 & 21,70 \\
1000 & 69,50 \\
16000 & 258,00
\end{tabular}

A partir de $q=3 \mathrm{o}$ environ, on a approximativement $i=\sqrt{q}$, ̀̀ un facteur constant près.

Ces résultats ont été soumis à des expériences de vérification, faites, les unes en comparant l'éclat du spectre continu donné par les flammes chargées de sodium à l'éclat de la raie, les autres en comparant l'éclat des deux raies $D_{1}$ et $D_{2}$ ou des deux raies violettes du rubidium. L'accord a été satisfaisant. De cet ensemble de vérifications nous devons conclure que notre hypothèse est conforme à la réalité, au moins pour les flammes colorées, dans lesquelles la vapeur métallique est toujours extrêmement diluée.

Dès lors, il est facile de calculer la transparence d'une flamme pour le rayonnement d'une autre, non identique : c'est ce que j'ai fait; j'en ai donné un Tableau à double entrée que l'on pourra trouver dans le Mémoire que j'ai inséré dans les Annales de Chimie et de Physique.

VI. Élargissement des raies. - La discussion des expériences précédeṇtes conduit à examiner le phénomène de l'élargissement des raies étroites. Il résulte de ces recherches que cet élargissement dépend seulement, à température constante, du produit $q$; il ne peut donc, en aucune façon, être pris pour mesure de la densité seule, comme on l'a dit quelquefois. L'élargissement des raies est d'ailleurs une conséquence nécessaire de ce fait, que le rayonnement de la vapeur est une fonction continue de la longueur d'onde, qui ne s'annule pas en dehors des raies, mais forme les fonds ou spectres continus, qui ne manquent jamais quand l'éclat est suffisant. Lorsque $q$ augmente, le milieu de la raie, formé de rayons qui ont atteint l'intensité maximum, ne peut pas augmenter d'éclat; l'éclat du fond qui entoure la raie s'accroît proportionnellement à $q$; entre ces deux extrèmes, on doit trouver tous les degrés intermédiaires, si la raie se raccorde avec le fond. L'augmentation d'éclat de la raie se fait donc pour ainsi dire latérale- 
ment, et sa largeur augmente. J'ai constaté directement que, la densité demeurant constante, les raies s'élargissent quand l'épaisseur augmente.

Cet élargissement ne peut ètre limité que par l'absence du fond. Comme celui-ci ne fait défaut dans aucune partie du spectre, l'épaisseur augmentant, la raie deviendra de plus en plus large, et la limite ne sera atteinte que lorsque le spectre sera entièrement continu et identique à celui que donnerait un corps noir porté à la température de la couche de vapeur. Il n'est donc pas exact de dire que les vapeurs peuvent être distinguées des solides par la nature de leur rayonnement, ceux-ci donnant un spectre continu et celles-là un spectre discontinu; cela n'est vrai que pour des vapeurs en couche peu épaisse ou peu dense, et cette observation me paraît importante pour les applications de l'analyse spectrale à l'Astronomie physique.

VII. Analyse quantitative. - Enfin, j'ai fait un assez grand nombre d'expériences dans le but d'examiner si les méthodes précédentes seraient applicables aux recherches d'analyse quantitative. Il en est résulté que, des solutions très étendues étant pulvérisées et entrainées en même quantité, la nature de l'acide du sel employé n'influe pas sur l'éclat du spectre avec les sels de sodium et de lithium quelles que fussent la richesse et la composition de ces solutions. Mais il n'en est pas de même pour ceux de calcium et de strontium. Enfin la quantité de vapeur colorée que renferme la flamme croît moins vite que la quantité de sel qu'entraìne le mélange : ce qui revient à dire que les réactions qui ont lieu dans la flamme dépendent de cette même quantité. Ce n'est qu'après une étude complète de ces phénomènes qu'on pourrait procéder à des analyses quantitatives.

Je me suis occupé aussi de l'influence de la température, mais ce sujet, assez complexe, exige de nouvelles recherches et les résultats déjà obtenus ne peuvent être résumés ici; on les trouvera dans le Mémoire original. 